\title{
Predictors of Optimum Uptake of Intermittent Presumptive Treatment of Malaria During Pregnancy Among Women at Navakholo Sub-County, Kakamega County - Kenya
}

\author{
Joseph Ndovoyo $^{1,3} \quad$ John Arudo $^{1} \quad$ Mary Kipmerewo $^{2} \quad$ Victor Mukaka $^{1} \quad$ Sharon Kosgey $^{1}$ \\ 1.Department of Clinical Nursing and Health Informatics, \\ Masinde Muliro University of Science and Technology, P.O BOX 190-50100- Kakamega - Kenya \\ 2. Department of Midwifery, Reproductive Health and Child Health, \\ Masinde Muliro University of Science and Technology, P.O BOX 190-50100- Kakamega - Kenya \\ 3. Department of Medical Services, \\ Kakamega County General Teaching \& Referral Hospital, P.O.Box 59-50100-Kakamega -Kenya
}

\begin{abstract}
Introduction. Malaria in pregnancy is associated with high incidences of maternal and neonatal mortality in malaria endemic regions. World Health Organization recommends Intermittent Presumptive treatment of malaria in pregnancy with Sulfadoxine-Pyrimethamine (IPTp-SP). It is recommended that every pregnant woman receives at least three doses administered one month apart up to the time of delivery. Despite increased antenatal clinic attendance and concerted efforts to address known barriers to uptake of malaria preventive measures in Navakholo Sub-County, uptake of three or more IPTp-SP doses in the Sub-County has remained low.

Objective. This study aimed at determining predictors of optimum uptake of intermittent presumptive treatment of malaria in pregnancy among women in Navakholo Sub-County.

Methodology. This was a cross sectional study using mixed methods of data collection. The study was carried out in Navakholo Sub-County, Kakamega County, Kenya. Multistage cluster sampling method was employed to attain sample size $(n=608)$. Data was analyzed using descriptive statistics, bivariate and multivariate logistic regression while thematic analysis was used for qualitative data. Strength of association between independent variables and dependent variables was measured using odd ratio and $\mathrm{p} \leq 0.05$ used to reject null hypothesis of no association between independent variables and the main outcome which was the uptake of optimum doses of IPTp-SP.

Results. Out of the 587 participants, 294(50.1\%) took optimum doses (three or more doses) of IPTp-SP, 248(42.2\%) took IPTp-SP partially (one-two doses) and 45 (7.7\%) did not take any dose. The following variables were statistically significantly associated with uptake of optimum doses of IPTp-SP: having attained secondary level of education and above ( $\mathrm{OR}=0.6,95 \% \mathrm{CI} 0.4-0.98, p=0.01)$; distance to health facility ( $\mathrm{OR}=0.2,95 \% \mathrm{CI} 0.06$ $0.8, p=0.02)$; perception that SP drugs are not safe during pregnancy $(\mathrm{OR}=7.3,95 \% \mathrm{CI} 1.5-35.7, p=<0.01)$; opening of health facilities daily ( $\mathrm{OR}=161.8,95 \% \mathrm{CI} 29.5-885.7 p<0.0001)$ and giving clients return dates (OR $=21.2,95 \%$ CI 7.9-56.5, $p=<0.0001)$.

Conclusion: Key factors that determine optimum uptake of IPTp-SP in the study area are: having attained at least secondary level of education; perceived safety of SP drugs; distance to health facility; opening of health facility daily and giving of return dates to clients.

Recommendation: - Community awareness through health education to increase awareness on the risks of malaria in pregnancy and safety of SPs in pregnancy. The study further recommends that the daily opening of facilities within the study area, ensure return dates are given at every visit and introduction of mobile clinics to those who are staying far away from the nearest health facility.
\end{abstract}

Keywords; IPTp-SP, Malaria in Pregnancy, Optimum uptake of IPTp-SP doses, Navakholo Sub-County.

DOI: $10.7176 / \mathrm{JHMN} / 60-08$

Publication date:March $31^{\text {st }} 2019$

\subsection{Introduction}

Malaria in pregnancy (MiP) contributes significantly to global maternal mortality in Sub-Saharan Africa. In 2015, malaria was the third most common cause of mortality among women of reproductive age in Africa. Malaria was estimated to have been responsible for more than 400,000 cases of maternal anemia and approximately $15 \%$ of maternal deaths globally. Unfortunately, the women who are most vulnerable to malaria are often the least protected against it. MiP also poses a significant threat to newborns because it can cause spontaneous abortion, stillbirth, premature delivery, low birth weight and neonatal mortality (Malaria Health Task Force, 2018).

A lot of efforts have been put in place to try and eradicate malaria infection. One of the aims of Roll Back Malaria (RBM) program that was adopted following the Abuja Declaration in 2000, was to ensure that by 2010, $80 \%$ of pregnant women would be protected from malaria. It was also anticipated that universal coverage would 
have been achieved by 2015 through prophylaxis with Sulfadoxine 500mg - Pyrimethamine $25 \mathrm{mg}$ (SP) and LongLasting Insecticidal Nets (LLINs) as key interventions (Gifty, 2010; Akoria, 2014). IPTp-SP is often associated with several benefits, among which are reduction in the risk of maternal anaemia and improvement of the outcomes of the fetus and neonatal life (WHO, 2012).

Several factors have been associated with uptake of IPTp-SP. In a study carried out in Tanzania, uptake of IPTp-SP was low due to uncertainty expressed by pregnant women on the safety of SP drugs in pregnancy. Uptake of IPTp-SP was further worsened when given to be swallowed at home (Kibusi, 2015). Similar findings were reported by Mbonye et al., (2006) who found that expectant mothers in Uganda were concerned with side effects anti-malarial drugs pose to themselves and their unborn babies. In addition, study findings from National Malaria Control Program (2014), identified client's socio-demographic factors such as age, marital status, level of education, parity, religion, occupational status and residence playing a major role in determining the uptake of IPTp-SP among pregnant women.

Kibusi, (2015) reported that among the gravid women at risk of missing IPTp-SP were young women aged 15-19 years as they were found to be starting their ANC visits late and hence low uptake of IPTp-SP. Higher uptake was associated with those in age bracket of 30-39 years. In Burkina Faso, Gies (2009) noted that ANC attendance increased with increase in age. A study conducted by Mutulei, (2013) found out that $82.2 \%$ of women in Bungoma, Kenya who had accessed two or more doses of IPTp-SP were married. In a related study carried out in Malawi, it was noted that the motivating factors for taking IPTp-SP included support from husbands, the desire to protect their children and themselves from malaria infection and the related complications (Mpanga et al., 2014). According to Mwandama et al., (2013), higher level of education is associated with the uptake of two or more doses of IPTp-SP. In a study that was done in Kenya, uptake of IPTp-SP was significantly associated with a woman's educational status (Mutulei, 2013). Similarly, other studies have reported that low levels of IPTp-SP uptake were associated with low levels of education (Agaba, 2013; Kibusi 2015). Employment also influenced optimum uptake of IPTp-SP as was found out in a study conducted in Korle-Bu Teaching Hospital in Accra (Ghana). The findings revealed that most of the women (80\%) not using IPTp-SP were of low income compared with $62.9 \%$ of those using (De Allegri et al., 2011). A study conducted in Juba, Southern Sudan, reported significant association between income and uptake of both ITN and IPTp-SP (Napoleon et al., 2011). Uptake of IPTp-SP was more likely among respondents with lower income than those with higher income. A woman's parity was equally found to be associated with optimum uptake of IPTp-SP. In a study where 142 women of different parities of between para 1+0 and para 4+0 were interviewed, uptake of IPTp-SP was significantly associated with multiparity (Mutulei, 2013). Multiparous women tend to meet the focused antenatal care (FANC) requirements of at least attaining 4 visits before delivery time. According to Iliyasu (2012), most of the pregnant women in Urban Kano in Northern Nigeria who were para two and above started their ANC visits in the $2^{\text {nd }}$ trimester (14-24 weeks' gestation) unlike the primigravidae who commonly started in their $3^{\text {rd }}$ trimester (28-32 weeks' gestation) who did not manage to attain the required number of minimum of 4 visits. In a study that was done in Bungoma, where most of the respondents (75\%) were Christians and Muslims (15\%), it revealed that the uptake of IPTp-SP and religious affiliation were not significantly associated (Mutulei, 2013). Despite high antenatal clinic attendance and increased efforts to address known barriers to the uptake of malaria preventive measures in the study area, uptake of 3 or more IPTp-SP doses in Navakholo Sub-County has remained low. The current uptake of IPTp-SP doses in the Sub-County is $40 \%$ short of the national target of $80 \%$ (DHIS $_{2}, 2017$; NMS, 2009-2017) and slightly above that of the entire Kakamega county that stands at $20 \%$ (KDHS, 2014). Therefore, to scale up the uptake of IPTp$\mathrm{SP}$, there is need to determine the predictors of optimum uptake of IPTp-SP in the study area.

\subsection{Methodology}

\subsection{Study Design}

This was a cross sectional analytical study design using mixed methods for data collection.

\subsection{Study setting}

The study was carried out in Navakholo Sub-County (see Appendix V). The main ethnic groups residing in this area are the Banyala and Batsotso whose dialects are Kinyala and Kitsotso respectively. The Sub-County falls within the malaria-endemic zone on the eco-epidemiological map of Kenya and malaria risk class of $20 \%$ or more (Mutulei, 2013). Health facilities in the Sub-County includes Navakholo Sub-County Hospital, three public health centres (Kharanda, Sivilie and Bushiri) and eight government dispensaries (Namirama, Buchangu, Sisokhe, Budonga, Butingo, Eshihongo, Ematiha and Kisembe). The Sub-County has two Faith Based health facilities, namely: Lutaso and Ingotse Dispensaries both of which offer ANC services including administration of Directly Observed Therapy (DOT) of IPTp-SP (DHIS- Navakholo Sub-County unpublished report, 2016). There are 13 community health units where Community Health Volunteers (CHV) through community health strategy enhances access to health care under the supervision of a Community Health Extension Worker (CHEW). Each CHV visits households assigned to them once every month where they provide pregnant women with haematenics, dewormers 
and health education. They also advise on immunization of their children and refer pregnant mothers to the health care facilities whenever they feel it is necessary, basically monitoring the health of the household members.

\subsection{Study population}

The study population comprised of women who had delivered within the last twelve months, and hence were expected to have sought ANC services, including IPTp-SP within the study area during the study period.

\subsection{Sampling technique}

Multistage sampling method was used. Stage one involved listing of the wards and random selection of three wards out of the five was done. In the second stage, three Sub-Locations were randomly selected out of the eight. Finally, households with post-delivery women were listed from each of the villages in the three sub-locations. Simple random sampling was used to select the 608 respondents based on the sampling frame which was compiled for each village. The 3 health facilities where Key Informant Interviews (KII) were done were purposively selected based on the maternal health services they offered. The facilities included one sub-county hospital, one public health centre and one faith-based dispensary. Four Focus Group Discussions (FGDs) of between 6-7 individuals per group were conducted one in each of the wards except for Bunyala West where 2 FGDs were done because of its relatively larger population of 38,407 (1 at Sidikho Chief's camp and 1 at Lusumu primary school\}, the rest was one in each ward, i.e. 1 at Namirama Friend's Church in Bunyala East ward, and 1 at Nambacha Market in Bunyala Central Ward.

\subsection{Eligibility criteria}

\subsubsection{Inclusion criteria}

Women who had delivered within the preceding twelve months and resident of Navakholo for the past six months or more before the study.

\subsubsection{Exclusion Criteria}

Women on Co-trimoxazole prophylaxis, those with history of allergy to sulfa drugs and non-residents.

\subsection{Sample Size}

The researcher used the "Modified EPI" Formula to estimate the minimum sample size. $\mathrm{n}=\mathrm{D} * \mathrm{Z}^{2 *} \mathrm{pq} / \mathrm{e}^{2}$

Where $\mathrm{n}=$ sample size, $\mathrm{D}=$ Design factor $(1.5), \mathrm{Z}=$ confidence interval (1.96), $\mathrm{p}=$ proportion of those taking optimum IPTp - SP doses $(40 \%), q=$ proportion of those not taking optimum doses of IPTp-SP $(60 \%)$ and e = margin of error $(0.05)$. The researcher added $10 \%$ of the sample size to cater for non-response. With these parameters, the computation yielded a sample size of 608 women.

\subsection{Research Instruments}

For quantitative data collection, the researcher modified and adopted a questionnaire that was used by Atasige, et al., (2016) and Ameh et al., (2016) in two studies conducted in a rural northern Ghana-Gushegu District and Cross River State in Nigeria, respectively.

\subsubsection{Quantitative data collection tool}

The researcher used a structured questionnaire which was divided into the following three sections: Section 1. Client characteristics; Section 2. Health facility related factors; and Section 3. Health provider related factors. The questionnaire was in English language and translated into Kiswahili in order to ease its execution and standardize responses. The questionnaire was again back-translated into English to ensure correct translation and no loss of meaning for each question. The questionnaire was evaluated to increase its validity and reliability. The instrument was pre-tested in Lurambi Sub-county which was outside the study area. The reliability of the instrument was relatively high (Cronbach's $\alpha=0.7$ ).

\subsubsection{Qualitative data collection tool}

Qualitative data was collected using focus group discussion (FGD) and key informant interview (KII). A total of four FGDs were conducted in each Ward with each group having 6-7 participants who delivered within the last 12 months. The FGD interview guide questions focused on knowledge, attitude and practice on IPTp-SP. For KII, their interview guide included questions on health provider and health facility related factors that influence optimum uptake of IPTp-SP doses. KII targeted the in-charges of MCH/FP departments in three health facilities, one in each ward.

\subsection{Data Collection Procedures}

The researcher conducted a three-days training for 7 research assistants (RAs) who were engaged in collection of quantitative data from the participants. The training focused on how to identify eligible respondents, interview techniques and ethical issues. The RAs comprised of 2 Kenya Registered Community Health Nurses (KRCHN), 
1 Nutritionist, 3 Community Health Volunteers CHVs) and 1 Supply Chain Management Officer (SCMO). Qualitative data was collected by the Principle Researcher. Data collection was undertaken from October to November 2017.

\subsection{Data Analysis.}

Quantitative data were entered, coded, cleaned and analyzed using Statistical Package for Social Sciences (SPSS) software version 22. Proportions were calculated using total number of respondents and denominator calculated based on the total number of respondents. Differences in the proportions were determined by calculating the confidence interval and the summary chi square statistic using Mantel Haenszel stratified cross-tabulations. pvalues of $\leq 0.05$ were regarded as statistically significant. Differences in the associations between categorical variables were assessed using Logistic Regression analysis. The Logistic Regression model was fitted to compare the association of optimum uptake of IPTp-SP and client characteristics (demographic and socio-economic factors), as well as health provider and health facility factors. The dependent variable was optimum uptake of IPTp-SP (three or more doses).

\subsection{Ethical considerations}

Approval to carry out the study was obtained from Institutional Ethics Review Committee (IERC) of Masinde Muliro University of Science and Technology. Further permission was sort from the County Administration. Authority to conduct the survey was granted by the National Commission for Science Technology and Innovation (NACOSTI).

Before commencing data collection, in order to introduce the project and answer any questions and concerns requiring clarifications, the researcher conducted an information session with service providers and community gate keepers. During interviews, study participants were taken through general questions about uptake of IPTp-SP with a view of identifying factors associated with optimum uptake of SP for prevention of malaria during pregnancy. These questions did not pose any risks to the participants.

Research assistants were especially cognizant of the importance of informing respondents at the various sites about the study and the purpose of the research and assuring them that their information would be kept confidential and anonymous. The researcher ensured that oral and written consent were obtained from the respondents before being interviewed. Participants were assured of confidentiality and that their participation was voluntary and that they were free to opt out at any stage without loss of any benefits. Therefore, all interviews were conducted within the respondent's home and in a private area but respecting the cultural norms. If the respondent indicated that she was uncomfortable holding the interview at that point, the interview was then shifted at another location of the interviewee's preference and convenience.

\subsection{Results}

\subsection{Background characteristics of the respondents.}

A total of 608 women participated in the study. Twenty-one (3.5\%) of the questionnaires that were incomplete due to missing data of key variables were excluded from data analysis leaving a total of 587 questionnaires that were analyzed. Table 1 shows socio-demographic characteristics of the respondents who were interviewed. The mean age was 26.8 years with a standard deviation of 6.1 years. The age range was between 15 to 50 years. Most of the respondents were in the age category of $25-34(47.4 \%)$ and $15-24(40.6 \%)$ years, respectively. Majority $(91.8 \%)$ were married or living with a partner. More than two-thirds (70.5\%) had attained primary level of education compared with $25 \%$ who had completed secondary education. Most of the respondents (55\%) were farmers with more than one-quarter (28.6\%) being housewives. Nearly two-thirds (63.2\%) were protestants compared to $22.8 \%$ who were Catholics. 
Table 1 Background characteristics of respondents

\begin{tabular}{|c|c|c|c|}
\hline Variable & Categories & $\mathrm{N}$ & $\%$ \\
\hline \multirow[t]{4}{*}{ Age groups (in years) } & $15-24$ & 238 & 40.6 \\
\hline & $25-34$ & 278 & 47.4 \\
\hline & $35-44$ & 69 & 11.7 \\
\hline & $\geq 45$ & 2 & 0.3 \\
\hline \multicolumn{2}{|c|}{ Mean age \pm SD (Range) in years } & \multicolumn{2}{|c|}{$26.8 \pm 6.1(15.0-50.0)$} \\
\hline \multirow[t]{5}{*}{ Marital status } & Single & 43 & 7.3 \\
\hline & Married & 539 & 91.8 \\
\hline & Divorced & 1 & 0.2 \\
\hline & Widow & 1 & 0.2 \\
\hline & Separated & 3 & 0.5 \\
\hline \multirow[t]{4}{*}{ Level of education } & None & 6 & 1.0 \\
\hline & Primary & 414 & 70.5 \\
\hline & Secondary & 147 & 25.0 \\
\hline & Tertiary & 20 & 3.4 \\
\hline \multirow[t]{5}{*}{ Occupation } & Housewife & 168 & 28.6 \\
\hline & Farmer & 323 & 55.0 \\
\hline & Employed & 16 & 2.7 \\
\hline & Self-employed/Business & 61 & 10.4 \\
\hline & Student & 19 & 3.2 \\
\hline \multirow{4}{*}{ Religion } & Catholic & 134 & 22.8 \\
\hline & Protestant & 371 & 63.2 \\
\hline & Muslim & 79 & 13.5 \\
\hline & Atheist & 3 & 0.5 \\
\hline
\end{tabular}

\subsubsection{Client characteristics associated with uptake of IPTp-SP}

Table 2 presents client characteristics that are associated with optimum uptake of IPTp-SP. Women who were protestants were one and a half times more likely to receive optimal doses of IPTp-SP than those who belonged to other religious groups such as Catholics and Muslims, among others (OR: 1.6; 95\% CI: $1.1-2.2 ; \mathrm{p}=0.01)$. The number of previous pregnancies was significantly associated with optimal uptake of IPTp-SP. Women who had less than 4 previous pregnancies were two-fold more likely to receive optimal doses of IPTp-SP compared with their counterparts who had higher number of previous pregnancies (OR: $2.0 ; 95 \%$ CI: $1.3-3.2 ; \mathrm{p}=0.002$ ). Similarly, women who had less than 4 previous deliveries were about two times more likely to receive at least three or more doses of IPTp-SP in contrast to those who had higher number of deliveries (OR: 1.7; 95\%CI: 1.1 $2.8 ; \mathrm{p}=0.02)$. Uptake was lowest among clients who had attained secondary school education and above compared with those who had primary or no education, although the association was marginal (OR: $0.7 ; 95 \%$ CI: $0.5-1.0$; $\mathrm{p}=0.06)$. 
Table 2 Client characteristics associated with uptake of IPTp-SP

\begin{tabular}{|c|c|c|c|c|c|c|}
\hline \multirow[t]{2}{*}{ Risk factor } & \multirow[b]{2}{*}{$\mathrm{N}$} & \multicolumn{2}{|c|}{ Uptake of IPTp-SP } & \multirow{2}{*}{$\begin{array}{l}\text { Overall } \\
\text { OR }\end{array}$} & \multirow[t]{2}{*}{$95 \% \mathrm{CI}$} & \multirow{2}{*}{$\begin{array}{l}\text { Significance } \\
\text { level } \\
\text { (p value) }\end{array}$} \\
\hline & & $\begin{array}{l}\geq 3 \text { doses of } \\
\text { IPTp-SP }\end{array}$ & $<3$ doses of IPTp-SP & & & \\
\hline \multicolumn{7}{|l|}{ Age group: } \\
\hline $30-39$ years & 152 & 55.3 & 44.7 & \multirow[t]{2}{*}{1.1} & \multirow[t]{2}{*}{$0.7-1.5$} & \multirow[t]{2}{*}{0.7} \\
\hline$<30$ or $>=40$ years & 435 & 53.8 & 46.2 & & & \\
\hline \multicolumn{7}{|l|}{ Marital status: } \\
\hline Married & 539 & 54.7 & 45.3 & \multirow[t]{2}{*}{1.3} & \multirow[t]{2}{*}{$0.7-2.3$} & \multirow[t]{2}{*}{0.4} \\
\hline Not married & 48 & 47.9 & 52.1 & & & \\
\hline \multicolumn{7}{|l|}{ Level of education: } \\
\hline $\begin{array}{l}\text { At least secondary } \\
\text { school }\end{array}$ & 165 & 47.9 & 52.1 & \multirow[t]{2}{*}{0.7} & \multirow[t]{2}{*}{$0.5-1.0$} & \multirow[t]{2}{*}{0.06} \\
\hline $\begin{array}{l}\text { Primary school or } \\
\text { none }\end{array}$ & 422 & 56.6 & 43.4 & & & \\
\hline \multicolumn{7}{|l|}{ Occupation: } \\
\hline Housewife & 168 & 50.6 & 49.4 & \multirow[t]{2}{*}{0.8} & \multirow[t]{2}{*}{$0.6-1.2$} & \multirow[t]{2}{*}{0.2} \\
\hline Employed & 419 & 55.6 & 44.4 & & & \\
\hline \multicolumn{7}{|l|}{ Religion: } \\
\hline Protestant & 371 & 58.2 & 41.8 & \multirow[t]{2}{*}{1.6} & \multirow[t]{2}{*}{$1.1-2.2$} & \multirow[t]{2}{*}{0.01} \\
\hline Others & 102 & 47.2 & 52.8 & & & \\
\hline \multicolumn{7}{|l|}{ Past obstetric hx: } \\
\hline $\begin{array}{ll}<4 & \text { previous } \\
\text { pregnancies } & \end{array}$ & 491 & 57.0 & 43.0 & \multirow[t]{2}{*}{2.0} & \multirow[t]{2}{*}{$1.3-3.2$} & \multirow[t]{2}{*}{0.002} \\
\hline $\begin{array}{ll}\geq 4 & \text { previous } \\
\text { pregnancies } & \\
\end{array}$ & 96 & 39.6 & 60.4 & & & \\
\hline \multicolumn{7}{|l|}{ Past delivery hx: } \\
\hline$<4$ previous deliveries & 502 & 56.2 & 43.8 & \multirow[t]{2}{*}{1.7} & \multirow[t]{2}{*}{$1.1-2.8$} & \multirow[t]{2}{*}{0.02} \\
\hline$\geq 4$ previous deliveries & 85 & 42.4 & 57.6 & & & \\
\hline
\end{tabular}

\subsection{Factors associated with access to health facility and uptake of IPTp-SP}

Table 3 presents findings on access to the nearest health facility and reveals a significant relationship between attendance of the clinic and uptake of optimal doses of IPTp-SP. Women who perceived that distance affects clinic attendance were $60 \%$ less likely to receive optimal doses unlike their colleagues who thought otherwise (OR: 0.4; 95\% CI: 0.2- 0.9; $\mathrm{p}=0.02)$. Women coming from areas where their nearest health facility opens every day were about 53 times more likely to receive optimal doses of IPTp-SP (OR: 52.8; 95\% CI: $16.5-169.2 ; \mathrm{p}<0.0001)$. The rest of the other facility-related factors had no significant association with the outcome (optimum uptake of IPTp-SP). Distance was mentioned as a hindrance to attend clinic as reported during the FGD in one of the sessions: I stay far away from the hospital and it is not possible for me to attend clinic frequently. Client's remark.

This was further confirmed by a second participant during FGD: "We find it difficult to reach the clinic because of distance. You have to hire a motorcycle at $£ 0.5$ for you to reach the health facility because it is not good for a pregnant woman to walk long distances. A remark from a Participant in Bunyala East Ward. 
Table 3: Factors associated with access to health facility and uptake of IPTp-SP

\begin{tabular}{|c|c|c|c|c|c|c|}
\hline \multirow[t]{2}{*}{ Risk factor } & \multirow[b]{2}{*}{$\mathrm{n}$} & \multicolumn{2}{|c|}{ Uptake of IPTp-SP } & \multirow{2}{*}{$\begin{array}{l}\text { Overall } \\
\text { OR }\end{array}$} & \multirow[t]{2}{*}{$95 \% \mathrm{CI}$} & \multirow{2}{*}{$\begin{array}{l}\text { Significance } \\
\text { level } \\
\text { (p value) }\end{array}$} \\
\hline & & $\begin{array}{l}\geq 3 \text { doses of } \\
\text { IPTp-SP }\end{array}$ & $\begin{array}{l}<3 \text { doses of } \\
\text { IPTp-SP }\end{array}$ & & & \\
\hline \multicolumn{7}{|l|}{$\begin{array}{l}\text { Access to health facility hindered } \\
\text { by geographical barriers: }\end{array}$} \\
\hline No & 411 & 54.0 & 46.0 & \multirow[t]{2}{*}{1.0} & \multirow[t]{2}{*}{$0.7-1.4$} & \multirow[t]{2}{*}{0.9} \\
\hline Yes & 176 & 54.6 & 45.6 & & & \\
\hline \multicolumn{7}{|l|}{$\begin{array}{l}\text { Type of health facility that is } \\
\text { nearest: }\end{array}$} \\
\hline Dispensary & 317 & 54.9 & 45.1 & \multirow[t]{2}{*}{1.1} & \multirow[t]{2}{*}{$0.8-1.5$} & \multirow[t]{2}{*}{0.7} \\
\hline Others & 270 & 53.3 & 46.7 & & & \\
\hline \multicolumn{7}{|l|}{$\begin{array}{l}\text { Time taken to reach nearest health } \\
\text { facility: }\end{array}$} \\
\hline$>1$ hour & 326 & 54.4 & 45.6 & \multirow[t]{2}{*}{1.0} & \multirow[t]{2}{*}{$0.7-1.4$} & \multirow[t]{2}{*}{0.9} \\
\hline$\leq 1$ hour & 261 & 54.0 & 46.0 & & & \\
\hline \multicolumn{7}{|l|}{ Distance affects clinic attendance: } \\
\hline Yes & 545 & 52.8 & 47.2 & \multirow[t]{2}{*}{0.4} & \multirow[t]{2}{*}{$0.2-0.9$} & \multirow[t]{2}{*}{0.02} \\
\hline No & 42 & 71.4 & 28.6 & & & \\
\hline \multicolumn{7}{|l|}{$\begin{array}{l}\text { Nearest health facility open every } \\
\text { day: }\end{array}$} \\
\hline Yes & 494 & 63.8 & 36.2 & \multirow[t]{2}{*}{52.8} & \multirow[t]{2}{*}{$16.5-169.2$} & \multirow[t]{2}{*}{$<0.0001$} \\
\hline No & 93 & 3.2 & 96.8 & & & \\
\hline
\end{tabular}

\subsection{Health provider related factors that affect uptake of optimum doses of IPTp-SP}

The relationship between provider related factors and optimal uptake of IPTp-SP was explored and presented in Table 4. Giving of return dates was a predictor of optimal uptake of IPTp-SP. Clients who were given return dates by health providers during their visits were 5-times more likely to receive the recommended optimal uptake of at least three doses of IPTp-SP than those who were not given return dates (OR: 4.7; 95\% CI: $2.6-8.6 ; \mathrm{p}<0.0001$ ).

Table 4: Health Provider factors associated with uptake of IPTp- SP

\begin{tabular}{|c|c|c|c|c|c|c|}
\hline \multirow[t]{2}{*}{ Risk factor } & \multirow[b]{2}{*}{$\mathbf{N}$} & \multicolumn{2}{|c|}{ Uptake of IPTp-SP } & \multirow{2}{*}{$\begin{array}{l}\text { Overall } \\
\text { OR }\end{array}$} & \multirow{2}{*}{$\begin{array}{l}95 \% \\
\text { CI }\end{array}$} & \multirow{2}{*}{$\begin{array}{l}\text { Significance } \\
\text { level ( } p \text { value) }\end{array}$} \\
\hline & & $\begin{array}{lr}\geq 3 & \text { doses } \\
\text { of } & \text { IPTp- } \\
\text { SP } & \end{array}$ & $\begin{array}{lr}<3 & \text { doses } \\
\text { of } & \text { IPTp- } \\
\text { SP } & \end{array}$ & & & \\
\hline \multicolumn{7}{|l|}{$\begin{array}{l}\text { Health education on pregnancy } \\
\text { provided to you by the health } \\
\text { provider during ANC visit clear and } \\
\text { concise. }\end{array}$} \\
\hline Yes & 547 & 54.1 & 45.9 & \multirow[t]{2}{*}{1.0} & \multirow{2}{*}{$\begin{array}{c}0.5- \\
1.8\end{array}$} & \multirow[t]{2}{*}{0.9} \\
\hline No & 40 & 55.0 & 45.0 & & & \\
\hline \multicolumn{7}{|l|}{ Staff ready to answer your concerns } \\
\hline Yes & 549 & 54.5 & 45.5 & \multirow[t]{2}{*}{1.2} & \multirow{2}{*}{$\begin{array}{c}0.6- \\
2.3\end{array}$} & \multirow[t]{2}{*}{0.6} \\
\hline No & 38 & 55.2 & 45.3 & & & \\
\hline \multicolumn{7}{|l|}{$\begin{array}{l}\text { Given return dates by the health } \\
\text { provider during your visits: }\end{array}$} \\
\hline Yes & 521 & 58.2 & 41.8 & \multirow[t]{2}{*}{4.7} & \multirow{2}{*}{$\begin{array}{c}2.6- \\
8.6\end{array}$} & \multirow[t]{2}{*}{$<0.0001$} \\
\hline No & 66 & 22.7 & 77.3 & & & \\
\hline
\end{tabular}

Although findings from quantitative study did not find any association between issuance of ANC card and uptake of IPTp-SP, results from KII revealed that expectant mothers feel secure when issued with ANC cards. Expectant women will therefore come to collect this cards and once they have acquired them, they stay at home and are unlikely to receive optimal doses of IPTp-SP. Such cards help them to avoid possible confrontation with the midwives as is supported by the following sentiments from one of the KII respondents:

Our clients are well informed. They know when faced with difficult labour at home, they will be brought to us. They know that the first thing we ask for is the ANC card. So they make sure at least they have registered and have a card just in case it becomes necessary. They say we quarrel them and may refuse to attend to them (Nurse at Sivilie Health Centre and Lutaso Dispensary giving a similar remark).

Support supervision is a key component in management in which staffs get motivated to provide evidence 
based healthcare. This study found out that focused supervision on IPTp-SP was lacking in the sampled health facilities and thus affecting optimum uptake of IPTp-SP as supported by the remarks from a facility in-charge as indicated below:

We are supervised by the County and sometimes Sub-County Health Management Teams but they have never come here to make a follow up on the issue of IPTp-SP in particular (Remarks of a Nurse in Navakholo Sub-County Hospital).

\subsection{Predictors of optimum uptake of IPTp-SP}

A multivariate logistic regression was performed to identify predictors of the optimum uptake of IPTp-SP as shown in table 5. Women who had attained at least secondary education were $40 \%$ less likely to receive optimum doses of IPTp-SP (OR: 0.6; 95\% CI: $0.4-0.9 ; \mathrm{p}=0.01$ ). The likelihood of uptake of optimum IPTp-SP doses was significantly higher among women who stated that the nearest health facility opens every day (OR: 161.8; 95\% CI: $29.5-885.7 ; p<0.0001)$. Women who were given return dates were also 21 times more likely to receive the recommended doses of IPTp-SP (OR: 21.2; 95\% CI: $7.9-56.5 ; \mathrm{p}<0.0001)$. Clients who reported that SP drugs are unsafe in pregnancy were 7 times more likely to take optimum doses of IPTp-SP as those who had indicated that they are safe. Those who believed that distance affected their clinic attendance were $80 \%$ less likely to receive optimum uptake of IPTp-SP doses (OR: 0.2; 95\% CI: $0.06-08 ; p=0.02)$. Being a protestant $(p=0.3)$, having had less than 4 previous pregnancies $(p=0.09)$ or deliveries $(p=0.7)$ and perception that IPTp-SP is recommended to be started after $1^{\text {st }}$ trimester had no significant association with the outcome.

Table 5: Multivariate logistic regression of predictors of the optimum uptake of IPTp-SP

\begin{tabular}{|l|c|c|c|}
\hline Risk factor & Overall OR & $95 \%$ CI & $\begin{array}{c}\text { Significance level } \\
(p \text { value })\end{array}$ \\
\hline Having attained at least secondary school education & 0.6 & $0.4-0.9$ & $\mathbf{0 . 0 1}$ \\
\hline Protestant & 1.2 & $0.8-1.9$ & 0.3 \\
\hline Less than 4 previous pregnancies & 2.9 & $0.9-10.0$ & 0.09 \\
\hline Less than 4 previous deliveries & 0.7 & $0.2-2.7$ & 0.7 \\
\hline Distance affects clinic attendance & 0.2 & $0.06-0.8$ & $\mathbf{0 . 0 2}$ \\
\hline SP drugs are not safe during pregnancy & 7.3 & $1.5-35.7$ & $\mathbf{0 . 0 1}$ \\
\hline IPTp-SP is recommended to be started after 1 1 $^{\text {st }}$ trimester: & 0.2 & $0.03-1.2$ & 0.08 \\
\hline Nearest health facility opens every day & 161.8 & $29.5-885.7$ & $<\mathbf{0 . 0 0 0 1}$ \\
\hline Given return dates by the health provider during your visits & 21.2 & $7.9-56.5$ & $<\mathbf{0 . 0 0 0 1}$ \\
\hline
\end{tabular}

\subsection{Discussion}

The objective of the study was to determine predictors of optimum uptake of IPTp-SP doses in Navakholo SubCounty. Among the socio-demographic factors that were significantly associated with optimal uptake of IPTp-SP were socio-economic factors such as level of education, religion, past obstetric history and past delivery history.

\subsection{Client characteristics}

Although education plays a critical role in the transformation of behavior and may positively influence decision making, our study findings show the contrary. Findings in this study shows that, women who had attained secondary level of education and above were less likely to benefit from optimal uptake of IPTp-SP as opposed to their counterparts with less education although the association was borderline. The finding is supported by other study results (Mubyazi, et al., 2014; Rumisha, et al., 2014) which showed that women with higher level of education were less likely to take IPTp-SP. However, our results are in contrast to that of a study conducted in Uganda and Tanzania (Mpungu, et al., 2008; Exavery, et al., 2014) which revealed that women with higher level of education were more likely to take three or more doses of IPTp-SP. The decreased optimal uptake of IPTp-SP as evidenced in the current study could be attributed to such women's busy schedules as in our study area (Olukaya, 2013). Most of the times women with secondary level of education are usually in employment and perhaps, are unlikely to get time to regularly attend antenatal clinic which is usually open only during working days as they are usually a way on duty.

In this survey, being a protestant was found to be significantly associated with optimal uptake of IPTp-SP as compared to other religions (Catholics and Muslims). In the recent past, there has been some disagreements in the Catholic leadership in Kenya and WHO regarding the safety of tetanus vaccine administration to pregnant women (Kenya Catholic Bishops press statement, 2015). There is a possibility that such church position regarding uptake of preventive medicine by pregnant mothers could have discouraged ANC clients who profess Catholic faith from taking optimal doses of IPTp-SP.

Another notable finding from this study which is similar to a study that was done in Nigeria by Olukoya $\&$ Adebiyi (2013) was that women with 4 or less previous deliveries were more likely to take optimum doses of 
IPTp-SP $(p=0.02)$. Women who had had more than four deliveries and had not suffered malaria, may not consider the importance of malaria in pregnancy and thus would downplay the risk. Again, this group could be shying away from coming to hospital to avoid some of the questions asked at the facilities noting the number of children one has had as they do not want to be seen as having delivered so many children. On the contrary, comparing uptake of IPTp-SP with the number of children one had, a study conducted by Akinyele (2009) found that women who were carrying pregnancy for the $1^{\text {st }}$ time, were found to be the highest consumers of IPTp-SP while the higher the number of children (four or more) the lower the uptake. In a related study done in Cameroon, Ngimuh, et al., (2016) found that uptake of IPTp-SP was high in multiparous women. He associated this to the fact that multigravid (para $2+0$ and above) women already knew the dangers of malaria in pregnancy since they had had a chance of receiving more health education on the importance of IPTp-SP and have complied hence increased its uptake.

While this study agrees with studies done in Tanzania that women between the ages of 20-34 are the highest consumers of IPTp-SP (Kibusi, 2015; Amon, 2014), the results were not statistically significant. Being in this age group was not associated with optimal uptake of IPTp-SP doses $(p=0.7)$. Probably, at this age $(20-34)$, most of the young women are getting into the stage of building their families through marriage and are eager to get healthy children from a malaria free antenatal period. Ngimuh et al., (2016) did a similar study in Cameroon and in their findings, most IPTp-SP users were in the $>25$-year-old age group. This may be associated with the fact that women in this age group are mature and most of them have attained a higher level of education and understand the importance of health care which includes receiving IPTp-SP to protect themselves and their unborn babies.

The findings in this study does not agree with the findings of Akinyele (2009), in which it was found that being married or staying with a partner, were associated with optimum uptake of IPTp-SP doses. This could be associated with the fact that couples tend to share views and encouragement. However, this study confirms the findings of Rumisha, et al., (2014) in which marital status was not a predictor of receiving more doses of IPTpSP. Sabin et al., (2010) also found insignificant association between marital status and uptake of optimum doses of IPTp-SP in Burkina Faso.

\subsection{Health Facility Related Factors that affect uptake of IPTp-SP}

Although in our study respondents were satisfied with the services offered, the findings from quantitative study did not find any association between issuance of ANC card and uptake of IPTp-SP. However, results from studies conducted in Mkuranga and Mufindi districts of Tanzania, Mubyazi, et al., (2014) found that pregnant women did not like the idea of sharing cups to swallow SP drugs in the clinic and that there was inadequate drinking water making them not attend ANC. This resulted in low uptake of IPTp-SP. In our study site, DOT policy is currently being implemented. The policy ensures that a pregnant woman takes SPs in the clinic under supervision. That notwithstanding, the approach seems to be forceful and disrespectful of the freedom of the clients as it does not allow them to opt out voluntarily. This may discourage pregnant women from attending ANC and eventually affecting uptake of IPTp-SP (Rumisha, et al., (2014).

Availability of SP drugs is critical to improve uptake of IPTp-SP. Most of the respondents (99.5\%) in this study reported that SP drugs were available as the drugs were supplied by a program supporting the Ministry of Health. Support from Health implementing partners in carrying out health care activities including procurement and distribution of SP drugs should be embraced as this ensures drug availability to increase uptake of IPTp-SP doses.

\subsection{Health Provider Related Factors that affect uptake of IPTp-SP}

This study found a significant association between ANC providers' action of giving return dates and uptake of IPTp-SP doses. Those clients who reported to have been given return dates by the health care provider were 21 times more likely to take optimal doses of IPTp-SP (OR 21.2, p $=<0.0001)$. This underscores the need to always remind clients that they need to come back for further assessments and care during pregnancy. This should be reinforced by noting return dates in their mother booklets for reference purpose and for easy remembrance.

In a similar study conducted by Sabina et al., (2006), confusion on the side of health workers about timing and spacing of SP doses and restriction of SP in early pregnancy or difficulty in correctly assessing gestational age of the woman contributes to missed dosing of IPTp-SP. Hurley, et al., (2016) also found that poor patient counseling about IPTp-SP and especially when DOT is not consistently practiced may impact negatively on uptake of IPT-SP. This usually resulted from patient-provider interactions that were fairly brief. Women were often instructed to take the pills with little or no explanation about their purpose.

Monitoring and supervision of ANC services ensures that lack of resources required for implementation of IPTp-SP program are promptly identified and addressed to ensure continuity of the program. This study found that there were no supervisory visits made to strengthen IPTp-SP program. Most of the staffs who were interviewed reported not having been visited. Lack of updates on IPTP-SP program hampered their service delivery. Indeed, supervision that targeted IPTp-SP services being offered was rarely done. Instead, County Health Management team who visited the facilities were interested in other services such as immunization, among others. The findings 
in this study concur with a study done by Doku et al (2016) in Tamale Metropolis in Ghana, in which monitoring of IPTp-SP was poor and that staffs were unable to track their IPT coverage leading to low uptake of optimal doses of IPTp-SP.

\subsection{Conclusion}

In conclusion, this study confirms that uptake of optimal doses of IPTp-SP in Navakholo Sub-County is determined by giving clients return dates and opening of health facilities daily. Similarly, level of education, distance to health facility and the client's perception on the safety of SP drugs in pregnancy are independently significantly associated with optimal uptake of IPTp-SP doses in the study area. Coverage rate of uptake of $\geq 3$ doses of IPTpSP $(50.1 \%)$ is still below the national recommendation of $80 \%$ with almost half of the pregnant women $(49.9 \%)$ having taken only one to two doses and $7.7 \%$ failing to have taken any doses. The success of IPTp-SP program will contribute to reduction of maternal and neonatal mortalities arising from malaria infection in pregnancy.

\subsection{Recommendations}

- Community awareness through health education should target those women who have at least secondary level of education and above so as to increase awareness on the risks of malaria in pregnancy and safety of SPs in pregnancy. Community based promotional activities through CHVs should be strengthened to increase health seeking behaviour for the pregnant women to be able to utilize ANC services.

- Health facilities should open on a daily basis and to run for 7 days a week so as to cater for the women who are employed and who may not get time during the weekdays to visit ANC.

- Mobile clinics in areas that are hard to reach due to poor road network should be considered.

- Ensure every client gets a return date. The county should employ more ANC health care workers so that staffs can have adequate time to engage their clients and provide full information including giving of return dates.

\section{Reference}

Atasige, A.S., Wurapa, F., Afari, E.A., Sackey, S.O., Malm, K.L. \& Nyarko, K.M. (2016). Factors influencing utilization of intermittent preventive treatment for pregnancy in Gushegu district, Ghana, 2013. Pan Afr Med J. 2016;25(Supp 1): 4 doi (10.11604/pamj.supp.2016.25.6.6169)

Agaba, P. (2013). Determinants of intermittent preventive treatment of malaria among pregnant women in Uganda. Princeton.edu/paper/131890

Akinyele, S.O., Falade, O.C., Ajayi, O.I., (2009). Knowledge and utilization of intermittent preventive treatment of malaria among pregnant women attending antenatal clinics in primary health care centers in rural southwest, Nigeria. BMC Pregnancy and Childbirth 2009 9:28. https://doi.org/10.1186/1471-2393-9-28.

Ameh, S., Owoaje, E., Oyo-Ita, A., Kabiru, C. W., Akpet, O. E., Etokidem, A., ... \& Ekpenyong, N. (2016). Barriers to and determinants of the use of intermittent preventive treatment of malaria in pregnancy in Cross River State, Nigeria: a cross-sectional study. BMC pregnancy and childbirth, 16(1), 99.

Atasige, S. A. (2014). Factors Influencing the Uptake of Intermittent Preventive Treatment of Malaria among Pregnant Women in the Gushegu District, Northern Region, Ghana (Doctoral dissertation, University of Ghana).

Doku, D.T., Zankawah, M. M., \& Adu-Gyamfi, A.B, (2016). Factors influencing dropout rate of intermittent preventive treatment of malaria during pregnancy. BMC research notes, 9(1), 460.DOI: 10.1186/s13104016-2265-2

De Allegri, M., Ridde, Louis, V.R., Sarker, M., Tiendrebeogo, J., Ye, M., ... \& Jahnn, A. (2011). Determinants of utilization of maternal care services after the reduction of user fees: a case study from rural Burkina Faso. Health Policy, 99(3), 210-218. District Health Information System 2 (DHIS 2,2017 ).

Exavery, A., Mbaruku, G., Mbuyita, S., Makemba, A., Kinyonge, I. P., \& H. Kweka (2014). Factors affecting uptake of optimal doses of Sulfadoxine Pyrimethamine for Intermittent Treatment of Malaria in Pregnancy in six districts of Tanzania. Malar J. 2014; 13:22 doi:10.1186/1475-2875-13-22 PMCID: PMC3898402

Gies, S., Coulibaly, S. O., Ky, C., Ouattara, F. T., Brabin, B. J., \& D’Alessandro, U. (2009). Community-based promotional campaign to improve uptake of intermittent preventive antimalarial treatment in pregnancy in Burkina Faso. The American journal of tropical medicine and hygiene, 80(3), 460-469.

Gifty, Dufie ANTWI (2010). Factors influencing the uptake of intermittent preventive treatment of malaria in pregnancy in Bosomtive district of Ghana

Iliyasu, Z., Gajida, A. U., Galadanci, H. S., Abubakar, I. S., Baba, A. S., Jibo, A. M., \& Aliyu, M.H. (2012). Adherence to intermittent preventive treatment for malaria in pregnancy in urban Kano, Northern Nigeria. Pathogens and GlobalHealth, 106(6), 323-329.

Irvine, M. H., Einarson, A., \& Bozzo, P. (2011). Prophylactic use of ant-malarial during pregnancy. Canadian Family Physician, 57(11), 1279-1281. 
Kenya Demographic and Health Survey KDHS, $(2014 ; 2017)$.

Kibusi, S. M., Kimunai, E., \& Hines, C. S. (2015). Predictors for uptake of intermittent preventive treatment of malaria in pregnancy (IPTp) in Tanzania. BMC public health, 15(1), 540.

Mbonye, A.K., Neema, S. \& Pascal, M. (2006). Treatment seeking practices for malaria in pregnancy among rural women in Mukono district, Uganda. Journal of Biosocial Science 38, p 221-237.

Mpanga, V., Maluwa, A., Kafulafula, U., Pindani, M., \& Bultemeier, K. (2014). Comprehension of Risk Factors of Malaria during Pregnancy among Pregnant Women Antenatal Care in Malawi. Open Journal of Nursing, 4(12), 896-905. doi: 10.4236/ojn.2014.412095.

Mubyanzi, G.M. \& Bloch, P. (2014). Psychosocial, behavioural and health system barriers to delivery and uptake of intermittent preventive treatment of malaria in pregnancy in Tanzania - viewpoints of service providers in Mkuranga and Mufindi districts. BMC Health Services Research201414:15. https://doi.org/10.1186/14726963-14-15.

Mutulei, A. C. N. E. (2013). Factors influencing the uptake of intermittent preventive treatment for malaria in pregnancy: Evidence from Bungoma East District, Kenya. American Journal of Public Health Research, 1(5), $110-123$.

Mwandama, D., Gutman, J., Wolkon, A., Luka, M., Jafali, J., Ali, D., ... \& Skarbinski, J. (2015). The use of intermittent preventive treatment in pregnancy and insecticide-treated bed nets for malaria prevention by women of child-bearing age in eight districts in Malawi. Malaria journal, 14(1),316.doi: 10.1186/s12936015-0840-y PMC4536790

Napoleon, R. P., Anyangu, A. S., Omolocan, J., \& Ongus, J. R. (2011). Preventingmalaria during pregnancy: factors determining the use of insecticide-treated bednets and intermittent preventive therapy in Juba. South Sudan Medical Journal, 4(2), 33-35

National Malaria Control Programme (NMCP), Kenya National Bureau of Statistics (KNBS), and ICF International. (2016). Kenya Malaria Indicator Survey 2015.

Ngimuh, L., Fokam, B.E., Achang-Kimbi, K.J. \& Wanji, S. (2016). Factors associated to the use of insecticide treated nets and intermittent preventive treatment for malaria control during pregnancy in Cameroon. The official journal of the Belgian Public Health Association201674:5. https://doi.org/10.1186/s136900160116-1

Olukoya, O.O. \& Adebiyi, O.A. (2013). Missed opportunities for intermittent preventive treatment for malaria in pregnancy in Nigeria: evidence from demographic and health survey in Nigeria. Ann Ibd. Pg. Med 2017. Vol.15, No.2 88-95

Ritchie, J., Lewis, J., Nicholls, C. \& Ormston, R. (Eds.). (2013). Qualitative research practice: A guide for social Science students and researchers. Sage.

Sabin, L. L., Rizal, A., Brooks, M. I., Singh, M. P., Tuchman, J., Wylie, B. J., ... \& Hamer, D. H. (2010). Attitudes, knowledge, and practices regarding malaria prevention and treatment among pregnant women in Eastern India. The American journal of tropical medicine and hygiene, 82(6), 1010-1016.doi 10.4269/ajtmh.2010.090339 PMCID:PMC2877404

Schantz-Dunn, J., \& Nour, N. M. (2009). Malaria and pregnancy: A global health perspective. Reviews in obstetrics and gynecology, 2(3), 186.

Webster, S., Lewis, J., \& Brown, A. (2013). Considerations in qualitative research. Qualitative research practice: A guide for social science students and researchers.

WHO (2012). Updated WHO Policy Recommendation. Intermittent Preventive Treatment of malaria in pregnancy using Sulfadoxine Pyrimethamine (IPTp SP).

Yaya, S., Olalekan, A.U., Amouzou, A. \& Bishwajit, G. (2018). Use of Intermittent preventive treatment among pregnant women in Sub-Saharan Africa: Evidence from Malaria indicator survey. Trop. Med. Infect. Dis.2018,318; doi:103390/tropialmed301001

\section{Acknowledgement}

I'm indebted to express my sincere gratitudes to my supervisors; Mr John Arudo, Dr Mary Kipmerewo, Mr Victor Mukaka and Sharon Kosgey for their professional guidance throughout the course of this study. I also wish to thank my colleagues both at my work place and at the university for their unwavering support and team spirit.

Mr John Arudo

Department of Clinical Nursing and Health Informatics, Masinde Muliro University of Science and Technology.

Dr, Mary Kipmerewo

Department of Reproductive Health, Midwifery and Child Health, Masinde Muliro University of Science and Technology. 